\title{
Westward Drift of Ionospheric Plasma Irregularities over a Low to Mid-latitude Transition Region in Indian Sector
}

\author{
Aashiq Hussain Bhat ${ }^{1}$, Bilal Ahmad Ganaie ${ }^{1}$, Thokuluwa K. Ramkumar², \\ Manzoor A Malik ${ }^{1, *}$ and P. Pavan Chaitanya ${ }^{2}$ \\ (1) Department of Physics, University of Kashmir, Hazratbal, Srinagar, Jammu and Kashmir-190006, India \\ (2) National Atmospheric Research Laboratory, Dept. of Space, Govt. of India, Gadanki, Tirupati, Andhra Pradesh 517112, India
}

Article history: received June 16, 2020; accepted May 28, 2021

\begin{abstract}
We report the observation of plasma depletions/plumes in the $\mathrm{F}$ region ionosphere over a low to middle latitude transition region in the Indian sector. The observation of these plasma depletions is based on the data obtained in May 2019 through the all-sky airglow CCD imager installed in the campus of University of Kashmir, Srinagar $\left(34.12^{\circ} \mathrm{N}, 74.83^{\circ} \mathrm{E}\right.$, magnetic latitude $25.91^{\circ} \mathrm{N}$ ). The depletions on the two consecutive nights of 05 and 06 May 2019 are aligned along the North-South (N-S) direction and drift westward. Several depletion bands along with some enhancement bands are seen in the 630-nm airglow images throughout the two nights. The observed structures show certain characteristics similar to Medium Scale Traveling Ionospheric Disturbances (MSTIDs) but these airglow features are not completely periodic. Further, in the observed depletion bands some East-West asymmetries are observed along with the structured tree-like branches of the airglow depletions. Some depletion bands even bifurcate leading to the inference that the structures are signatures of plasma irregularities rather than the usual MSTIDs observed in low-mid latitude transition region. The westward drift of the depletions especially during geomagnetic quiet times over this region makes this study significant since it offers a possible evidence that shows extension of spread $\mathrm{F}$ irregularities from the mid latitude region to the low-mid latitude transition region. In this paper, we point out some possible mechanisms related to the occurrence of plasma depletions at this region and their westward movement during geomagnetic quiet times.
\end{abstract}

Keywords: Airglow Imager; Plasma Irregularities; Geomagnetic Low-mid latitude transition region; Post-midnight MSTID; Atmospheric Gravity Waves.

\section{Introduction}

The frequently observed features in the F-region ionosphere are the Medium-scale traveling ionospheric disturbances (MSTIDs) over mid-latitudes and plasma bubbles (EPBs) in the equatorial and low geomagnetic latitudes. MSTIDs are typically wave-like structures driven by the atmospheric gravity waves and influence the 


\section{Aashiq Hussain Bhat et al.}

structuring of the plasma bubbles [Kelley, 2011]. The plasma irregularities at the equatorial ionosphere are generated through Rayleigh-Taylor instability (RTI), but are not confined to the equatorial region as these may extend both altitudinally and latitudinally during the generation [Makela, 2006] However, the consequences of these irregularities do not have to be limited to the equator as the density depletions caused by equatorial Spread-F (ESF) can extend to higher altitudes and to the equatorial anomaly latitudes. Ma and Maruyama [2006], for example, announced the discovery of a plasma bubble at $31^{\circ}$ magnetic latitude. Further, plasma density depletions were also observed by Haung et al. [2007] at magnetic latitudes of $40^{\circ}-50^{\circ}$.

In addition to MSTIDS and EPBs, although limited, there are evidences of generation of plasma irregularities at mid-latitude regions as well. Various measurement techniques such as ionosondes, radar, airglow imaging observations, and satellite-based measurements have been used over the time to investigate the occurrence, properties, and generation process of mid-latitude spread-F [Sivakandan et al. 2020 and references therein]. At middle latitudes, field aligned irregularities (FAIs) are a fascinating phenomenon associated with MSTIDs. Some studies indicate that the MSF is generally linked to TIDs and particularly with MSTIDs [e.g., Bowman \& Monro, 1988; Otsuka et al., 2009]. Since the post-midnight FAIs' zonal propagation velocities are similar to that of MSTIDs at middle latitudes, some post midnight FAIs may be mid-latitude FAIs simultaneously existing with MSTIDs [Otsuka, 2012] Taori et al. [2015] found EPBs and MSTIDs in the lower latitudes of the Indian sector at the same time, suggesting that MSTIDs may be one of their seeding mechanisms. Both the EPB and the MSF are capable of producing FAIs [Saito et al., 2008]. The EPBs tend to drift eastward, while the MSF structures tend to drift westward [Sivakandan et al. 2020] and even though EPBs can map up to mid-latitudes, the propagation path can be used to distinguish between EPBs and MSF. The gradient drift instability is thought to be the most likely cause of FAIs on scales less than $50 \mathrm{~km}$ [Kelley et al., 2004; Otsuka et al., 2009]. Sun et al. [2015] has mentioned that the polarisation electric fields associated with Es patches can effectively map along magnetic field lines to the airglow-depleted regions in the F layer, causing FAIs with the same scale size $(10-15 \mathrm{~km})$ as the Es patches. Large scale plasma irregularity with characteristic scale sizes over $100 \mathrm{~km}$, on the other hand, have received little attention. Further, in the geomagnetic low-mid latitude transition regions such studies are very limited. Therefore, the studies of these large scale FAIs in these regions and their correlations with gravity waves and MSTIDs are necessary. Furthermore, the position of the all sky imager in this study is intriguing because it is surrounded by Himalayan mountain ranges, where wind flows can produce a lot of lee waves, causing secondary instabilities in the upper atmosphere.

In this study, we provide an evidence of westward drifting plasma bubble depletion structures extending from mid-latitude regions towards the low-latitude regions. The observations provide an insight of the extension of plasma depletion bands generated in middle latitudes towards the transition region. The westward drift of plasma depletion bands and the presence of branching like structures in these depletions towards the geomagnetic transition region will also be discussed.

\section{Instrumentation and Image Data Processing}

\subsection{All-sky Airglow Imager (Installed at Srinagar, India)}

Many types of observations have been used to detect and characterize the nighttime MSTIDs and plasma irregularites in the earlier studies. One of the most important technique of observation of MSTIDs is the $630.0 \mathrm{~nm}$ airglow imaging which has shown valuable applications in revealing the 2-D structure of nighttime MSTIDs in recent years [Narayanan et al., 2014; Martinis et al., 2010; Otsuka et al., 2004; Shiokawa et al., 2003a; Garcia et al., 2000]. An all-sky imager mounted at the University of Kashmir (hereafter $\mathrm{KU})\left(34.12^{\circ} \mathrm{N}, 74.83^{\circ} \mathrm{E}\right.$; magnetic latitude: $25.91^{\circ} \mathrm{N}$ ) was used to collect the data used in this study. The data analyzed in this paper was collected during the month of May 2019 and the plasma depletion structures are observed on two consecutive nights of 05 and 06 May. On other nights of observation, no depletion bands are observed. Srinagar is considered as one of the relatively high latitude stations in the Indian sector and is near to the geomagnetic low to mid-latiude transition region. A broad band OH filter with a central wavelength of $840 \mathrm{~nm}$ notched at $866 \mathrm{~nm}$ and four narrow band filters: $557.7 \mathrm{~nm}$ OI emission, $630 \mathrm{~nm}$ OI emission, $866 \mathrm{~nm}$, and $868 \mathrm{~nm}$ are fitted in the filter wheel of this imager. At near infrared wavelengths, the etaloning effects are prominent for $866 \mathrm{~nm}$ and $868 \mathrm{~nm}$ filters [Ramkumar et al., 2021]. So the data of these filters are not presented here as it requires further treatment of received emission signals. For the images 
used in this analysis, the exposure times were 10, 100 and 100 second for the 857, 557.7 and $630 \mathrm{~nm}$ filters, respectively. As a result, the airglow images have a temporal resolution of approximately 4 minutes.

The average of around 30 minute image data is taken to recognise the presence of plasma irregularity and MSTID signatures in the OI $630 \mathrm{~nm}$ images, and then the average image is subtracted from each image of this 30 minute averaged data, resulting in residual images. On comparing the resulting images to the contrast enhanced images, it is seen that the wave structures in the residual images do not vary significantly from the contrast enhanced images, with the exception of background intensity variations. The benefit of subtracting this averaged image is that it accounts for the continuous instrumental noise as well as intensity effects like the van Rhijin effect [Narayanan et al., 2009]. In order to compensate for the van Rhijin effect, Garcia et al. [1997] proposed using the average image for flat fielding. The edges of alternate bright and dark bands can be easily identified using this analysis.

To convert the obtained image to real dimensions, the orientation of images is calibrated and pixel correspondences to spatial scales are identified at the assumed peak emission altitudes. In this analysis, we assumed that the peak altitude of the airglow emission layer was $250 \mathrm{~km}$, and that OI $630.0 \mathrm{~nm}$ emissions originated there. The spatial scales of the images processed in the manner described above are obtained through the calibration of image pixels using coordinate mapping, as described by Kubuta et al. [2001]. The images are given geographic scales as a result of this coordinate conversion. At $250 \mathrm{~km}$ altitude, the imager covers a circular distance of 1800 kilometres with a maximum field of view (FOV) of 180 degree. Because of the intensity effects due to nearby buildings, trees and street lights we have reduced the processing procedure at the time of geographic conversion of images for a limited FOV of 140 degree. With this cut off in FOV, at a peak emission layer altitude of $250 \mathrm{~km}$, it occupies a horizontal distance of around $1220 \mathrm{~km}$ [Bhat et al., 2021]. Figure 1 shows the example of a raw image and the processed image converted into geographic coordinates. The image used in this figure was obtained during the night of May 6, 2019, at 23:29 IST.

The spatial extension of the plasma irregularities is calculated both manually and from keograms. The two points are chosen at the ends of minimum intensity regions, say (xi, yi) and (xf, yf), so that the distance between them is given by

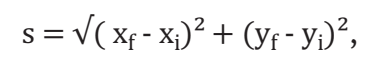

where "s" represents the horizontal extension of the plasma irregularity under consideration [Sivakandan et al., 2020].
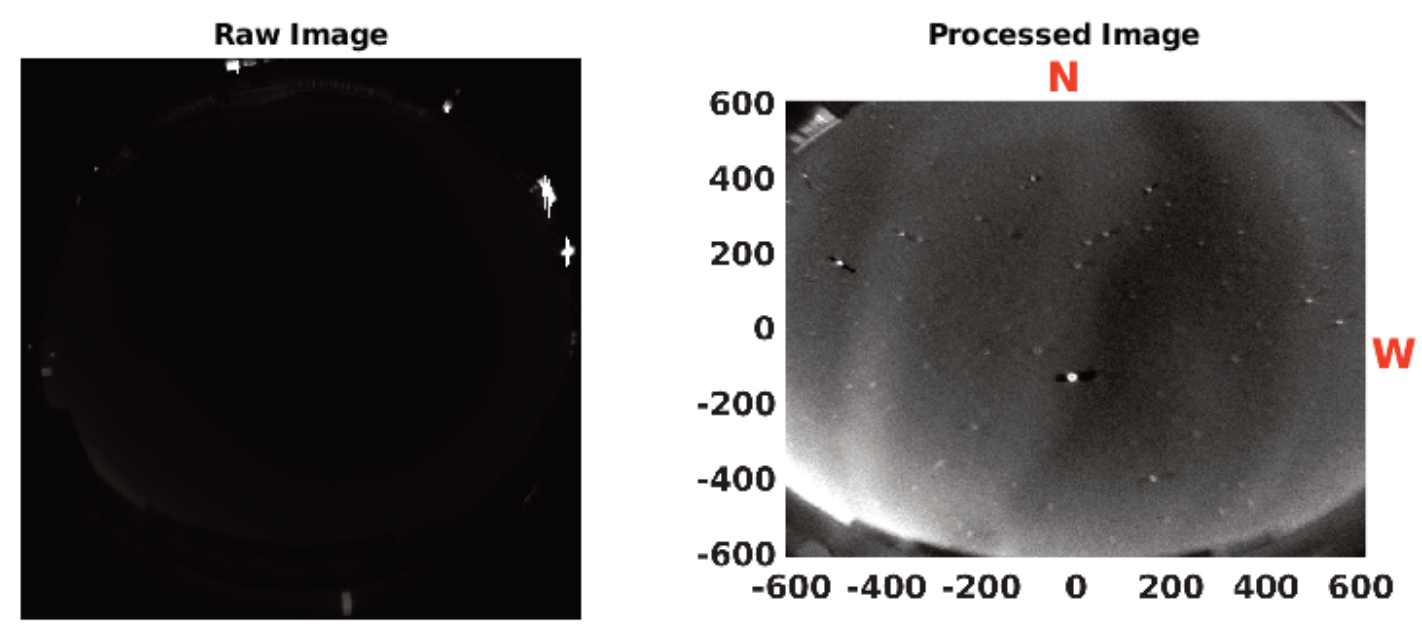

Figure 1. Example of $630 \mathrm{~nm}$ airglow image a) Raw image and b) Processed cropped image in geographic coordinates centered at the location of the all sky Imager. The image is flat fielded corrected for background brightness. The central point $(0,0)$ represents the zenith (overhead Srinagar), and the horizontal and vertical coordinates reflect distance in kilometres. The image shown is taken at 23:29 IST on 06 May 2019. Top of the images represent north and the right sides represent west. 


\section{Aashiq Hussain Bhat et al.}

The image data is also analysed by generating east-west (EW) and north-south (NS) keograms. The EW keograms are plotted by fixing latitude and varying longitudes while the NS keograms are constructed by fixing longitude and varying latitude. The keograms are built from the portion of the images enclosing a horizontal distance of 1220 $\mathrm{km}$ at an assumed altitude of $250 \mathrm{~km}$. The benefit of keogram analysis over individual images is that the zonal and meridional components of phase velocities can be determined separately. The keograms for the $630 \mathrm{~nm}$ image data for the nights of May 5 and 6 are shown in the following section. Both keograms and the manual method are used to measure westward zonal drift velocities.

\subsection{Gadanki lonospheric Radar Interferometry (GIRI; Installed at Gadanki, India)}

GIRI data is used to search for any signs of ionospheric disturbances in the equatorial or low latitudes during the nights in question. This $30 \mathrm{MHz}$ radar is installed at National Atmospheric Research Laboratory (NARL) in Gadanki which is one of the low latitude stations in India $\left(13.5^{\circ} \mathrm{N} ; 79.2^{\circ} \mathrm{E}\right.$, Magnetic latitude $\left.\sim 6.5^{\circ} \mathrm{N}\right)$. To get the detailed descriptions of this radar and its data processing one can refer to Patra et al. [2014].

\section{Results}

The observations of $630 \mathrm{~nm}$ airglow intensities were carried out during clear sky and moonless nights with an exposure time of $100 \mathrm{~s}$. In the present work, we have taken OI $630 \mathrm{~nm}$ image data from the month of May 2019. Specifically, we present the results of 05 and 06 May 2019 in which various ionospheric plasma irregularities are observed. The observations provide an insight of the extension of plasma depletion bands generated in middle latitudes towards the low-mid latitude transition region.

\subsection{May 05, 2019 Plasma Irregularity Events}

On the night of May 05, 2019, various depletion structures are observed with last one appearing as less contrasted structure compared to the earlier depletions. Figure 2 shows a set of images acquired on this night with time indicated on the top of every image in the Indian Standard Time format (IST). In the first image obtained through $630 \mathrm{~nm}$ filter at 21:25 IST (first few images not shown in the panel) a dark plasma depletion structure is observed from the North-East (NE) and propagating towards west. This dark plasma depletion structure is prominantly visible in the images from 21:40 IST onwards as shown in Figure 2. It appears with a bifurcation in between the depletion region or somewhat like a slingshot with two branching type structures as its two limbs. As the time progresses, the depletion band continues to propagate westward with average drift velocity of around $200 \mathrm{~ms}^{-1}$ for both the western and the eastern limbs. It may be noted that the longitudinal spatial extension of the western and eastern branches of this depletion structure are around $185 \mathrm{~km}$ each. Another depletion structure appears in the FOV of the imager from the NE side of the images at around 22:33 IST and grows in contrast as it continues to propagate westward. This second depletion structure appears with three branching type striations as is clearly visible in the images after 23:04 IST shown in the 2nd row of the images (Figure 2). The EW inter-depletion distance of the eastern branch decreases rapidly with its westward propagation and on average the total east-west inter-depletion extension of the eastern, central and western limbs are about $230 \mathrm{~km}, 185 \mathrm{~km}$ and $105 \mathrm{~km}$ respectively. The average zonal drift velocities of these branches are $160 \mathrm{~ms}^{-1}, 200 \mathrm{~ms}^{-1}$ and $116 \mathrm{~ms}^{-1}$ respectively. The western most branch of the depletion doesn't extend to much lower latitudes relative to its middle and eastern most branches. It means that the latitudinal extension of western branch of the depletion is lesser as compared to the other two branches. The two extended branches bifurcate at around 23:41 IST. In the image at 23:41 IST (Figure 2), it can be clearly seen that the two depletion branches appear as two separate propagating structures. Soon as the bifurcation occurs, a new depletion structure appears in the FOV from the same side as the earlier depletion structures. It has relatively much larger longitudinal extension (around $216 \mathrm{~km}$ ) compared to first two structures with average and zonal drift velocity of about $240 \mathrm{~m} / \mathrm{s}$. Further, this depleted structure is planer and has no visible branching regions associated with it unlike the earlier two observed depletion bands although its intensity of depletion is lesser as compared to its 
previous counterparts. In the processed airglow images, the contrast of this depletion structure diminishes with time because of the decrease in overall airglow intensity as the night progresses. Some MSTID bands in the later images are also seen whose details are presented in the next subsection.
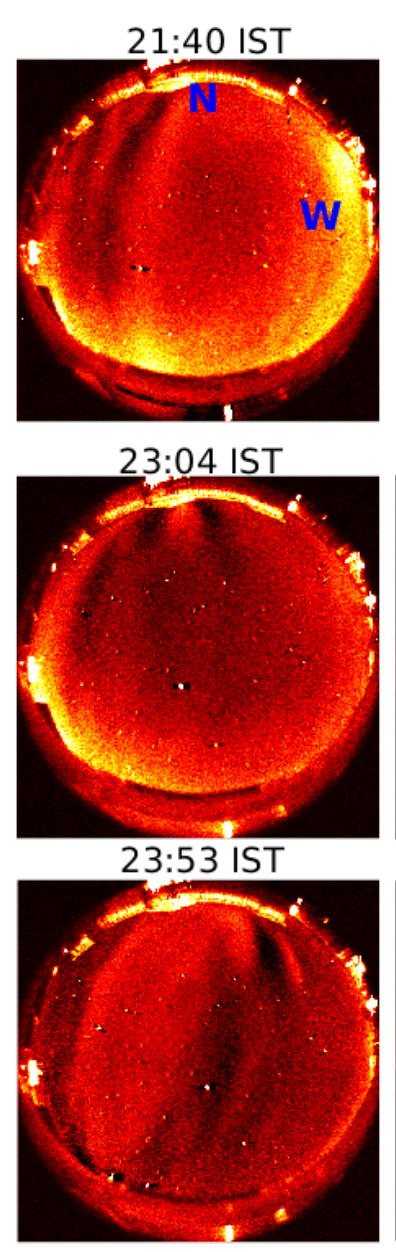

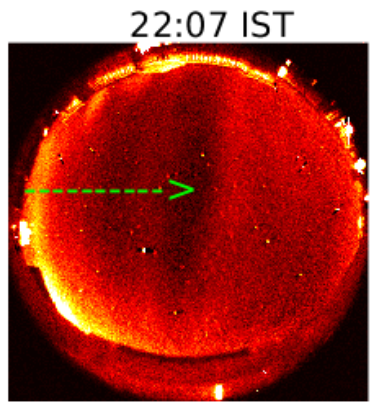

23:15 IST
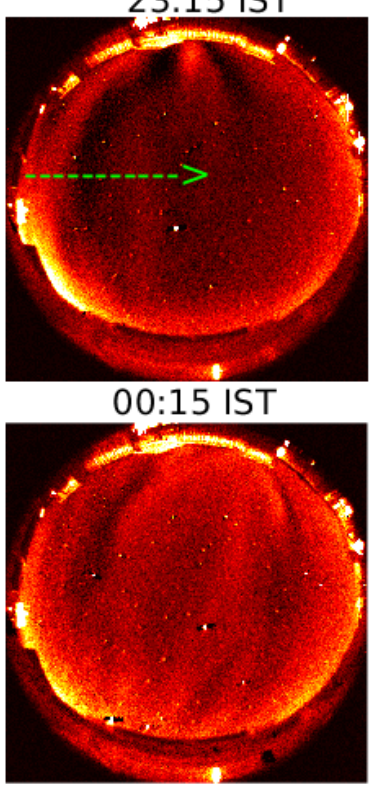

22:33 IST

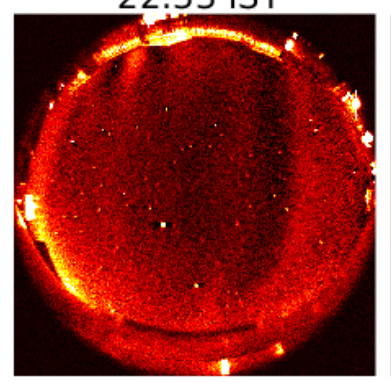

23:22 IST
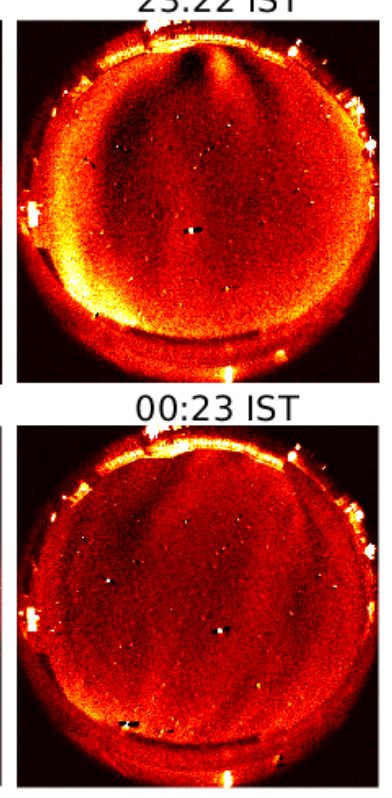

22:45 IST

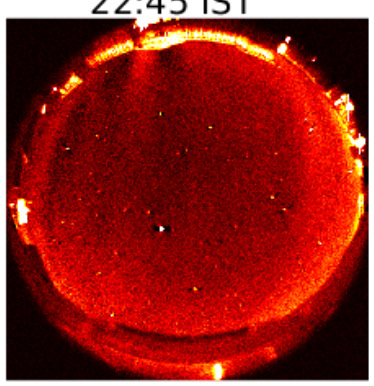

23:41 IST

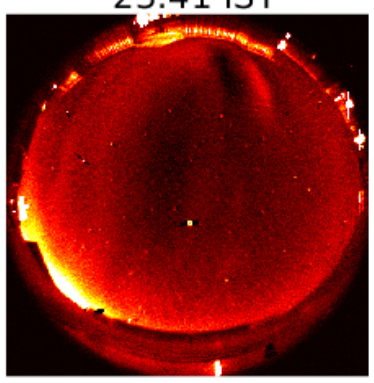

00:42 IST

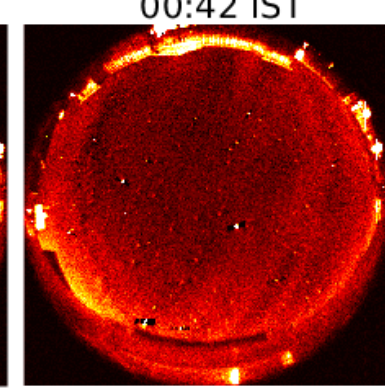

Figure 2. Time evolution of the irregularites observed from $630.0 \mathrm{~nm}$ night-glow emissions on 05 May, 2019. Raw images are subtracted from 30 minute time averaged images to produce the images. IST stands for Indian Standard Time in the images (Local Time).

In Figure 3, we present some of the images taken on 05 May showing the appearance of irregularites from the $\mathrm{NE}$ side of the imager. These are seen to extend latitudinally downwards (note the change of position indicated by green colored star) in addition to their westward drift. It indicates that the observed irregularities might be the extensions of mid-latitude generated irregularities towards the lower latitude regions.

The keograms for the images are constructed to visualize the time evolution of the observed airglow events and to calculate the drift velocity of the structures. For this, we have chosen the middle pixel regions of the images. The zonal (EW) and meridional (NS) keograms for the 05 May image data of $630 \mathrm{~nm}$ airglows are shown in Figures 4a and $4 \mathrm{~b}$ respectively. From these keograms, we can easily see the westward propagation of the depletion structures along with various branching regions. Moreover, from the meridional cut, it is seen that the structures are field aligned, the different intensity variations being parallel to the time axis. It can be noted that the last depletion band is a deep depleted planer structure without any bifurcating structures apparent in both the cuts. The various parameters associated to these depletion structures are summarized in table 1. 

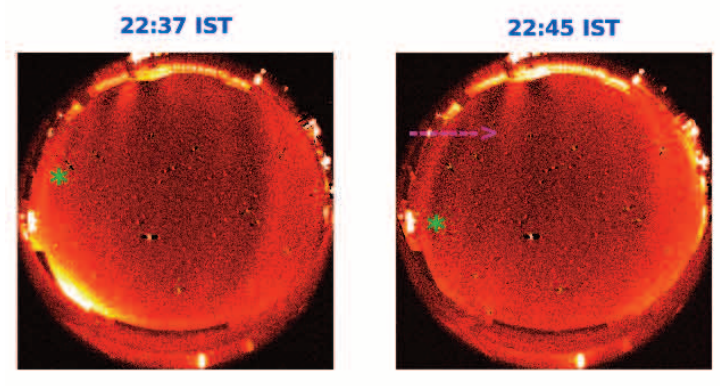

N
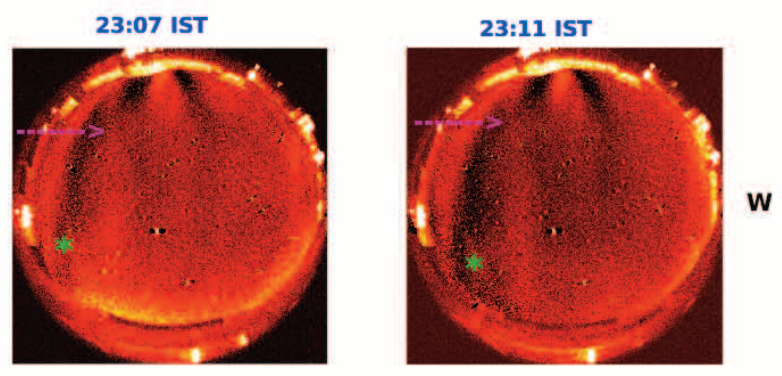

Figure 3. Extension of plasma irregularites towards lower latitudes during the night of 05 May, 2019. The green colored star shows the low-latitude extension of the plasma irregularites.

\begin{tabular}{cccccc} 
Event & $\begin{array}{c}\text { Observation } \\
\text { Time (IST) }\end{array}$ & $\begin{array}{c}\text { Average East-West } \\
\text { Interdepletion } \\
\text { Distance }(\mathbf{k m})^{*}\end{array}$ & $\begin{array}{c}\text { Average zonal } \\
\text { drift velocity } \\
\left(\mathbf{m s}^{-1}\right)^{*}\end{array}$ & $\begin{array}{c}\text { Number of } \\
\text { secondary } \\
\text { branching } \\
\text { structures }\end{array}$ & $\begin{array}{c}\text { Propagation } \\
\text { Direction }\end{array}$ \\
$\begin{array}{c}\text { Field Aligned } \\
\text { Irregularity }\end{array}$ & $21: 25-22: 45$ & 190 & 210 & 2 & Westward \\
\hline $\begin{array}{c}\text { Field Aligned } \\
\text { Irregularity }\end{array}$ & $22: 03-00: 27$ & 185 & 200 & 3 & Westward \\
\hline $\begin{array}{c}\text { Field Aligned } \\
\text { Irregularity }\end{array}$ & $23: 45-01: 46$ & 200 & 240 & 0 & Westward \\
\hline
\end{tabular}

* the spatial extension and velocity correspond to the major irregularity and not the branching regions.

Table 1. Parameters of the Observed Wave Events Observed on 05, May 2019.
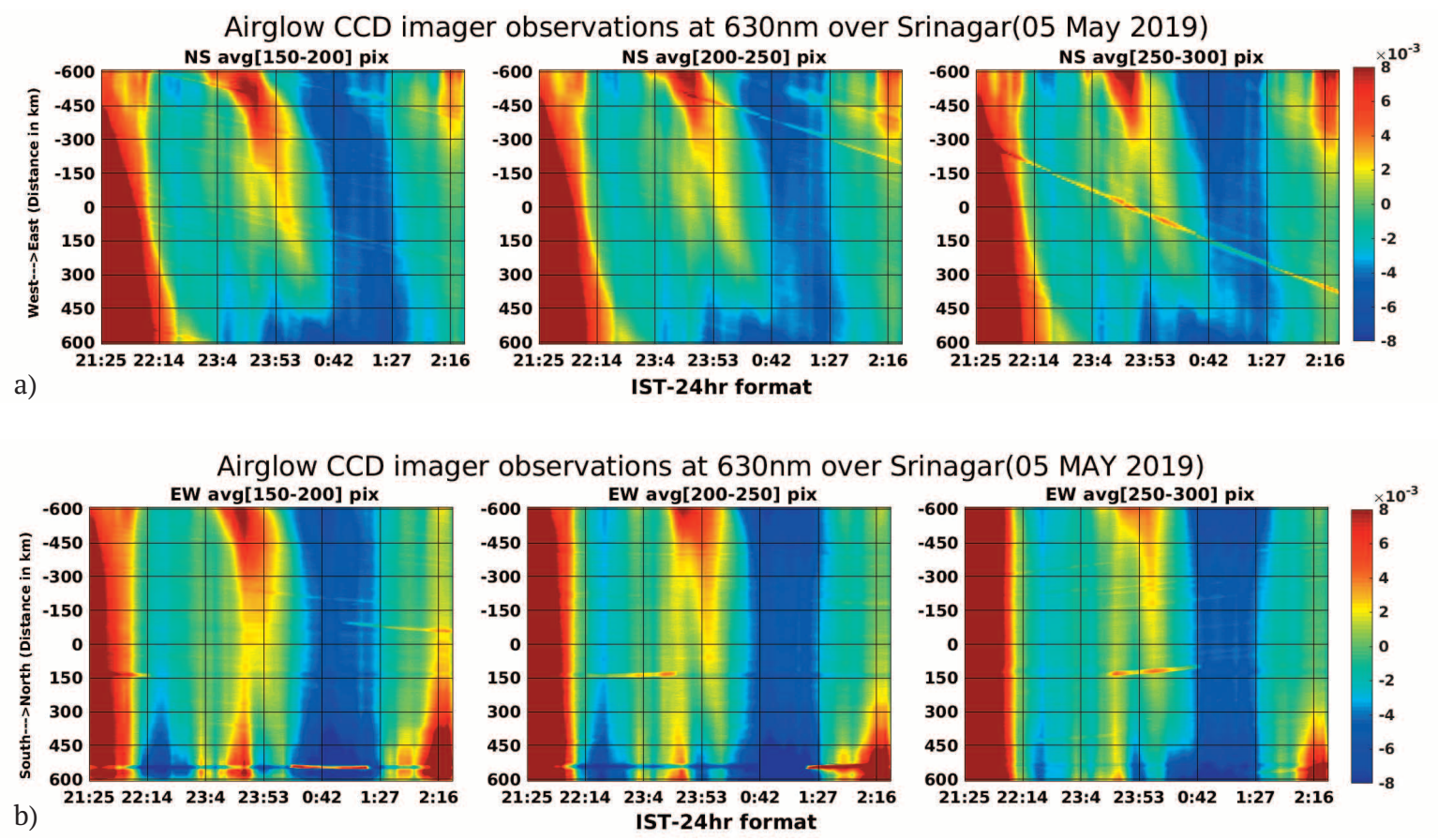

Figure 4. a) Zonal keogram of $630.0 \mathrm{~nm}$ night-glow emissions on 05 May, 2019 and b) Meridional keogram of $630.0 \mathrm{~nm}$ night-glow emissions on 05 May, 2019. 


\subsection{May 05, 2019 MSTID Event}

Near midnight, the MSTIDs are also observed and are seen in the lower latitude side of the images. These structures observed in the $630 \mathrm{~nm}$ images from 23:49 - 01:23 IST, move southwestward with an estimated horizontal wavelength of about $195 \mathrm{~km}$ and average phase velocities of $110 \mathrm{~ms}^{-1}$. The southwestward moving structures have NW to SE aligned phase fronts with time period around 30 minutes and lasted for about 90 minutes in the FOV of the imager. Based on observed parameters such as wavelength, time span, and propagating speed, the moving structures are categorised as MSTIDs. The wave like periodicity of these structures can be easily observed in the southern part of the images (Figure 5) but is not visible in the northern regions indicating their prominence in the lower latitude side of the location only. The coordinates in the images shown in Figure 5 represent distance in km with center $(0,0)$ representing overhead KU campus in Srinagar.

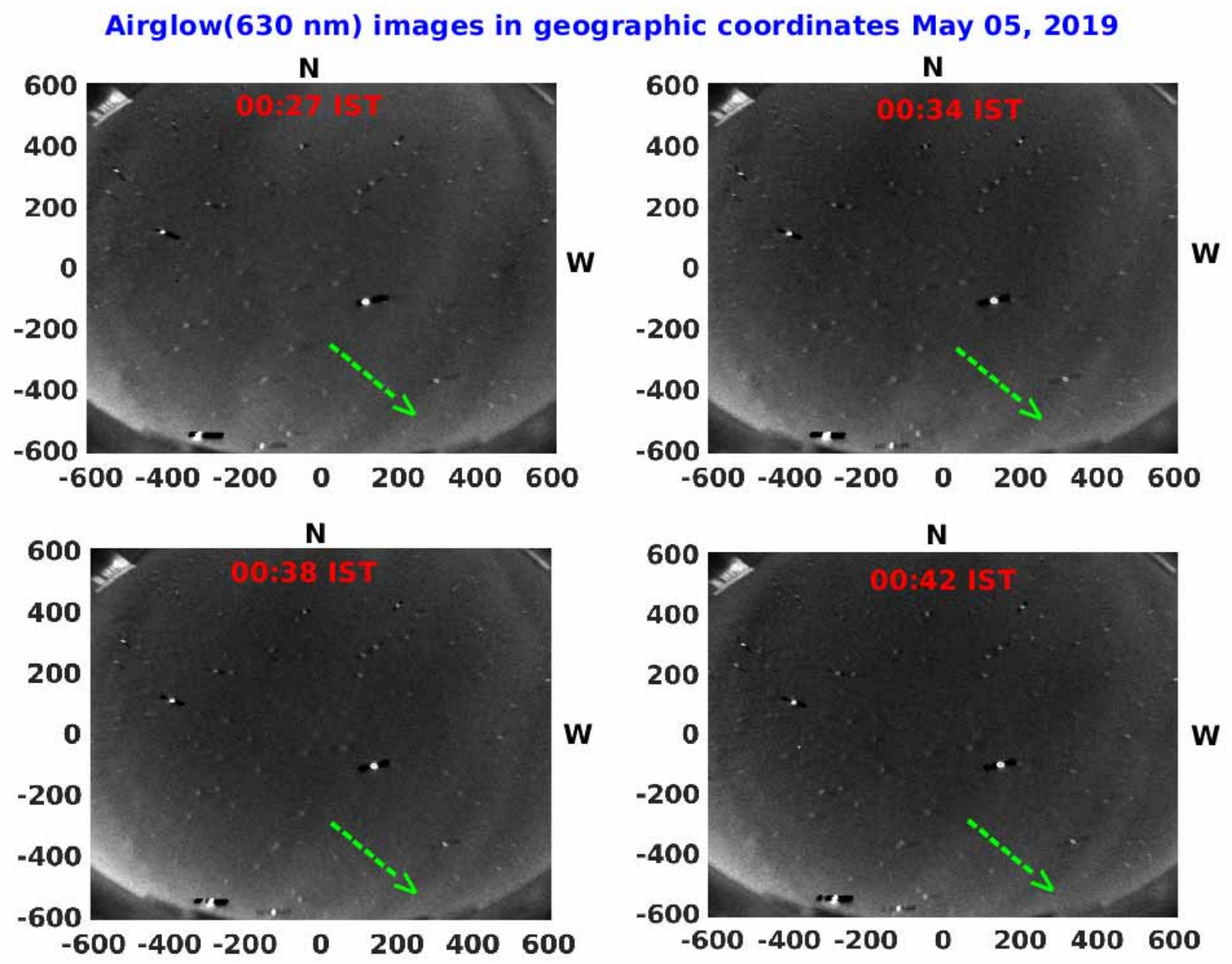

Figure 5. MSTID structures observed from $630.0 \mathrm{~nm}$ night-glow emissions on 05 May, 2019. The images are created by subtracting raw images from 30-minute time-averaged images and then converting them to geographic coordinates. IST in the images stands for Indian Standard Time (Local Time). Green arrows indicate the direction of propagation of MSTID.

\subsection{May 06, 2019 Plasma Irregularity Events}

The all sky imaging of $630 \mathrm{~nm}$ airglow observation on the night of May 06, 2019 began at 21:17 IST and in the initial period of about half an hour the northwestward moving passing clouds are recorded in the images making it impossible to notice any airglow structures for this period. After this half an hour time sky got clearer as the clouds 


\section{Aashiq Hussain Bhat et al.}

passed out of the FOV of the imager. When the clouds vanish from its field of view, a tree-like branched structure emerges, as seen in the first two panels of Figure 6. As can be seen on the top side of the images, these tree-like branching structures are part of FAI and extend from the middle latitude regions. The East-West spatial extension of this FAI is around $144 \mathrm{~km}$ and its central, eastern and western branching structures are around $85 \mathrm{~km}, 144 \mathrm{~km}$ and $126 \mathrm{~km}$ in width respectively. The average westward velocity of this irregularity is $160 \mathrm{~m} \mathrm{~s}^{-1}$. Around 22:40 IST, this depletion structure vanishes from the imager's field of view. In addition to this FAI, three other plasma depletion structures are also observed as the night progresses taking the total number of observed irregularity structures on the 06 May night to four. The second plasma depletion visible in the images from about $23 \mathrm{hrs}$ local time shows certain bifurcation features associated with it. The two plasma depletions that followed it did not have any branching structures. Hence, all the four irregularity structures have their own morphological features and other characteristics. The full time evolution of the events observed during the 06 May night is shown in Figure 6. The important parameters and other relevant features calculated/observed from the images are summarized in Table 2.
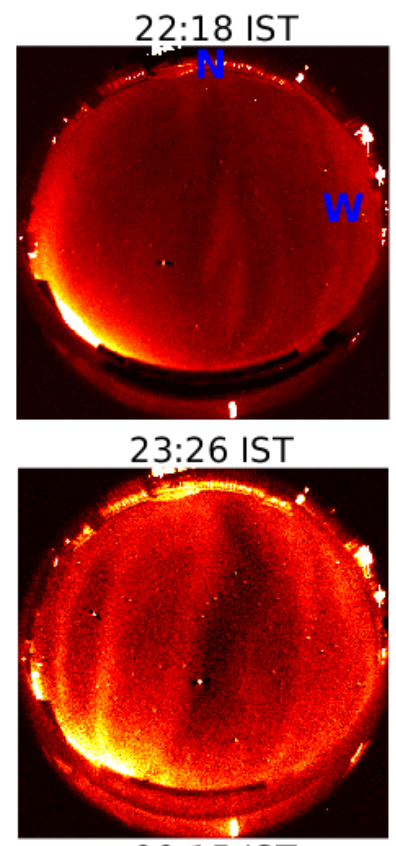

00:15 IST

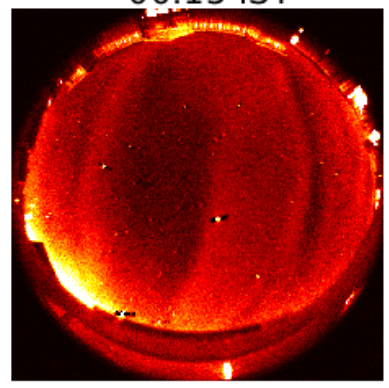

22:25 IST

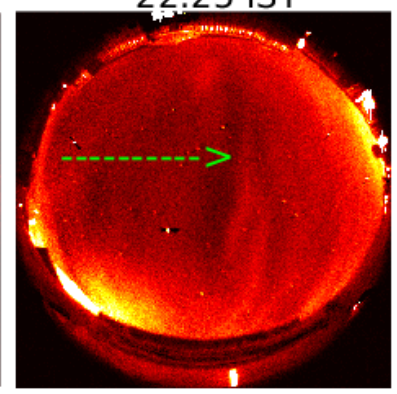

23:37 IST

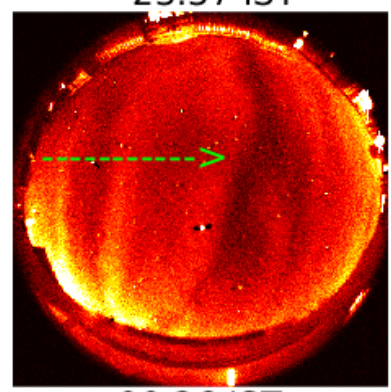

$00: 26$ IST

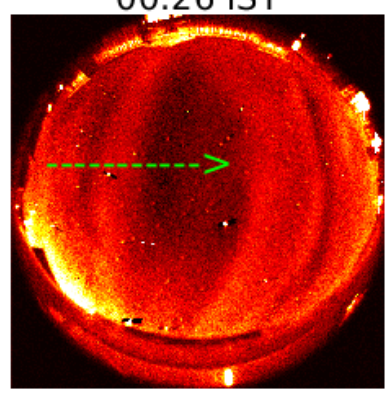

23:03 IST

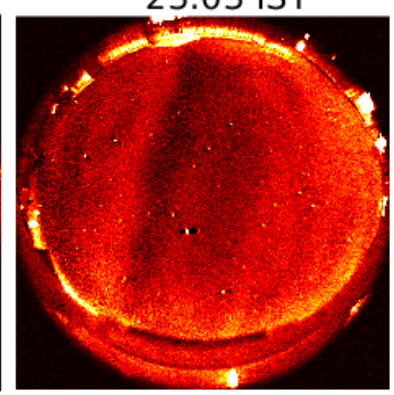

23:44 IST

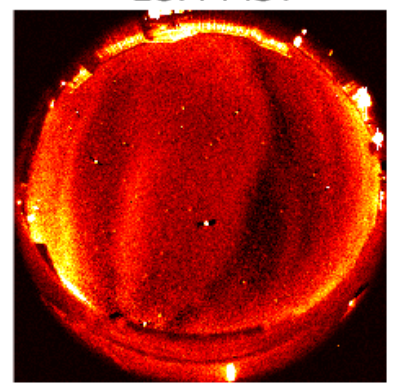

$00: 41$ IST

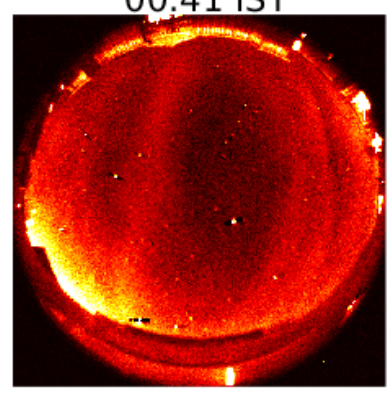

23:14 IST

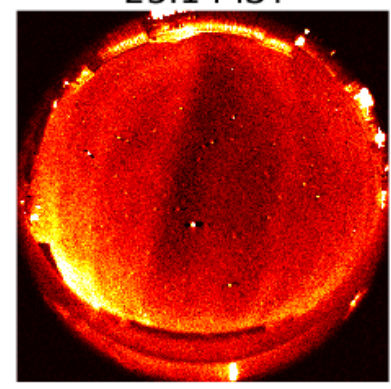

00:00 IST
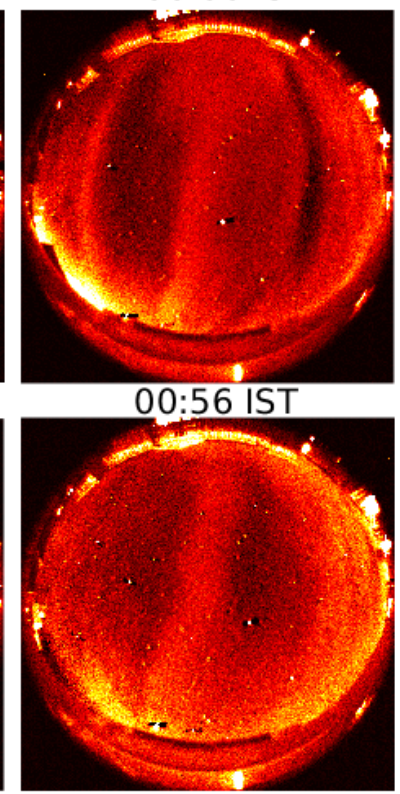

Figure 6. Time evolution of the plasma irregularity structures observed from $630.0 \mathrm{~nm}$ night-glow emissions on 06 May, 2019. Raw images are subtracted from 30 minute time averaged images to produce these images. IST in the images stands for Indian Standard Time (Local Time).

The examples of the different plasma irregularities observed during this night are shown in Figure 6 in the geographic coordinates. The morphology of the 2nd irregularity structure on May 06 night is similar to the first one on the 05 May night. It appears as a double branched structure just like a slingshot. The eastern wall of the irregularity gets more tilted towards east as the time progresses. This is evident from the 3rd image (23:29 IST) in Figure 7. The first irregularity is observed with no noticeable tilts unlike the other three irregularities which have eastward tilts. 


\begin{tabular}{cccccc} 
Event & $\begin{array}{c}\text { Observation } \\
\text { Time (IST) }\end{array}$ & $\begin{array}{c}\text { Average East- } \\
\text { West Spatial } \\
\text { Extension } \mathbf{( k m )}\end{array}$ & $\begin{array}{c}\text { Average zonal } \\
\text { drift velocity } \\
\left(\mathbf{m s}^{-1}\right)^{*}\end{array}$ & $\begin{array}{c}\text { Number of } \\
\text { visible } \\
\text { secondary } \\
\text { branching }\end{array}$ & $\begin{array}{c}\text { Propagation } \\
\text { Direction }\end{array}$ \\
$\begin{array}{c}\text { Field Aligned } \\
\text { Irregularity }\end{array}$ & $21: 51-23: 03$ & 144 & 160 & 3 & Westward \\
\hline $\begin{array}{c}\text { Field Aligned } \\
\text { Irregularity }\end{array}$ & $22: 36-00: 41$ & 288 & 130 & 2 & Westward \\
\hline $\begin{array}{c}\text { Field Aligned } \\
\text { Irregularity }\end{array}$ & $23: 18-01: 34$ & 468 & 190 & 0 & Westward \\
\hline $\begin{array}{l}\text { Field Aligned } \\
\text { Irregularity }\end{array}$ & $00: 22-02: 23$ & 360 & 170 & 0 & Westward \\
\hline * the spatial extension and velocity correspond to the major irregularity and not the branching regions.
\end{tabular}

Table 2. Parameters of the Observed Wave Events (06 May, 2019).

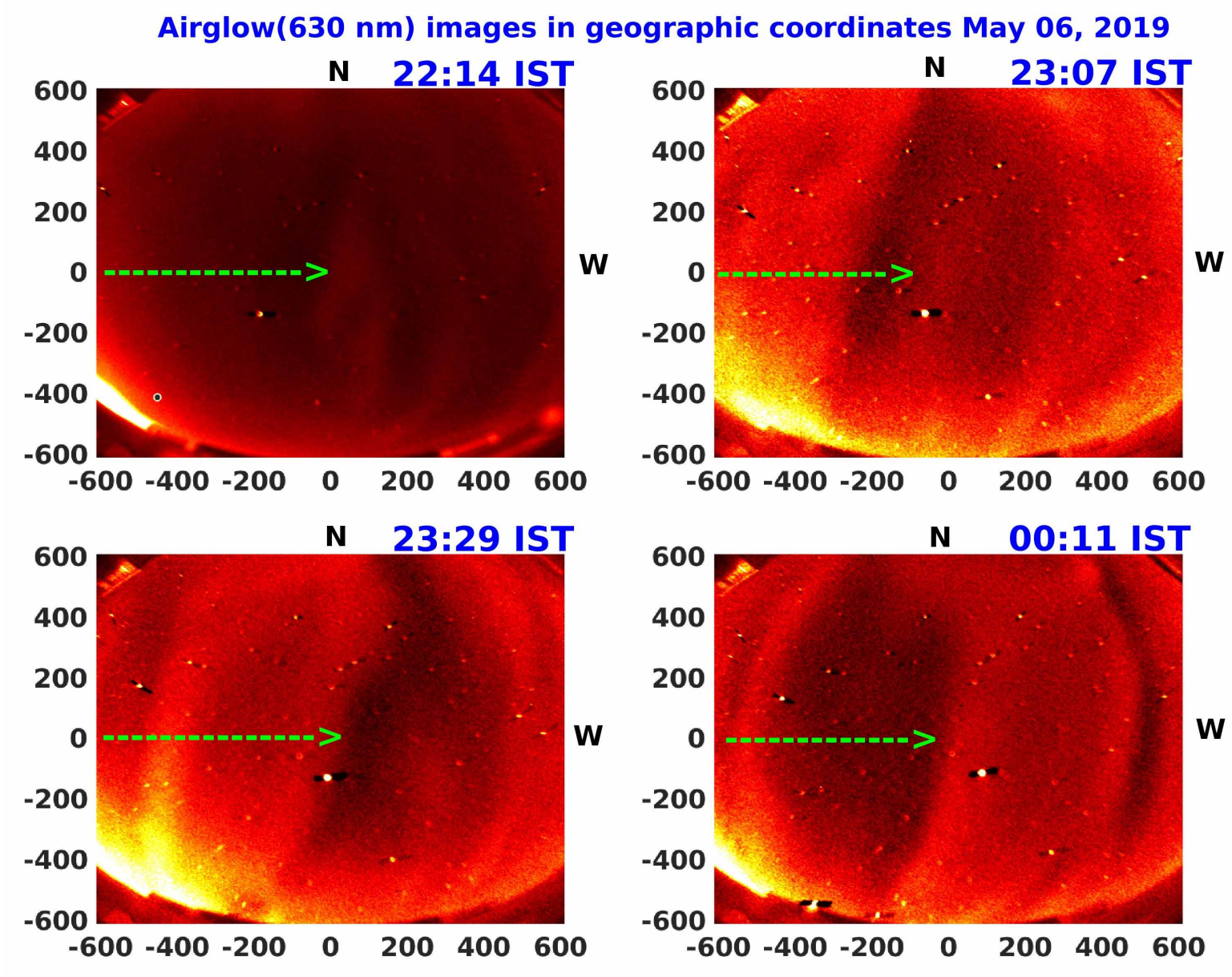

Figure 7. Examples of the processed images in geographic coordinates acquired on May 06, 2019 night. The images show four different irregularity structures at different times on the same night. The coordinates represent distance in $\mathrm{km}$ with center $(0,0)$ representing overhead KU campus in Srinagar. 


\section{Aashiq Hussain Bhat et al.}

Further, the other two depletion bands observed during and after the midnight time are deep depleted and planer structures with no branching regions. These planer irregularities are also having eastward tilts just like the branching type irregularities observed across the two nights. In both the nights, the irregularities observed before midnight have noticeable branching like structures while post mid-night irregularities appear with no such branching but planer structures. These observed structures show certain characteristics similar to MSTIDs but the structures are not completely periodic. Further, in the observed depletion bands some East-West asymmetries are observed along with the structured tree-like branches of the airglow depletions. Thus, in the present case the results we observe are plasma depletion structures with East-West asymmetries along with several tree like bifurcation striations which are not relevant to MSTIDs. Due to the presence of the bifurcating structures, alignment along geomagnetic field, significant depletion levels, East-West asymmetries and the westward drifts of these structures we infer that structures observed are field aligned plasma irregularities rather than the usual MSTIDs observed in mid-latitudes.

The zonal and meridional keograms for the 06 May night are shown in Figure 8a and 8b respectively. From the keograms, the time evolution of the observed irregularity structures is evident. A bright blob type of structure seems to be there in the keograms which can also be seen in the processed airglow images. The airglow intensity is much larger at these regions and is probably because of the plasma from depletion regions pushed towards these regions leading them to get intensified relative to background. Thus, plasma depletions and a large scale plasma blob are observed sailing together in the ionospheric region over Srinagar during the 06 May night.
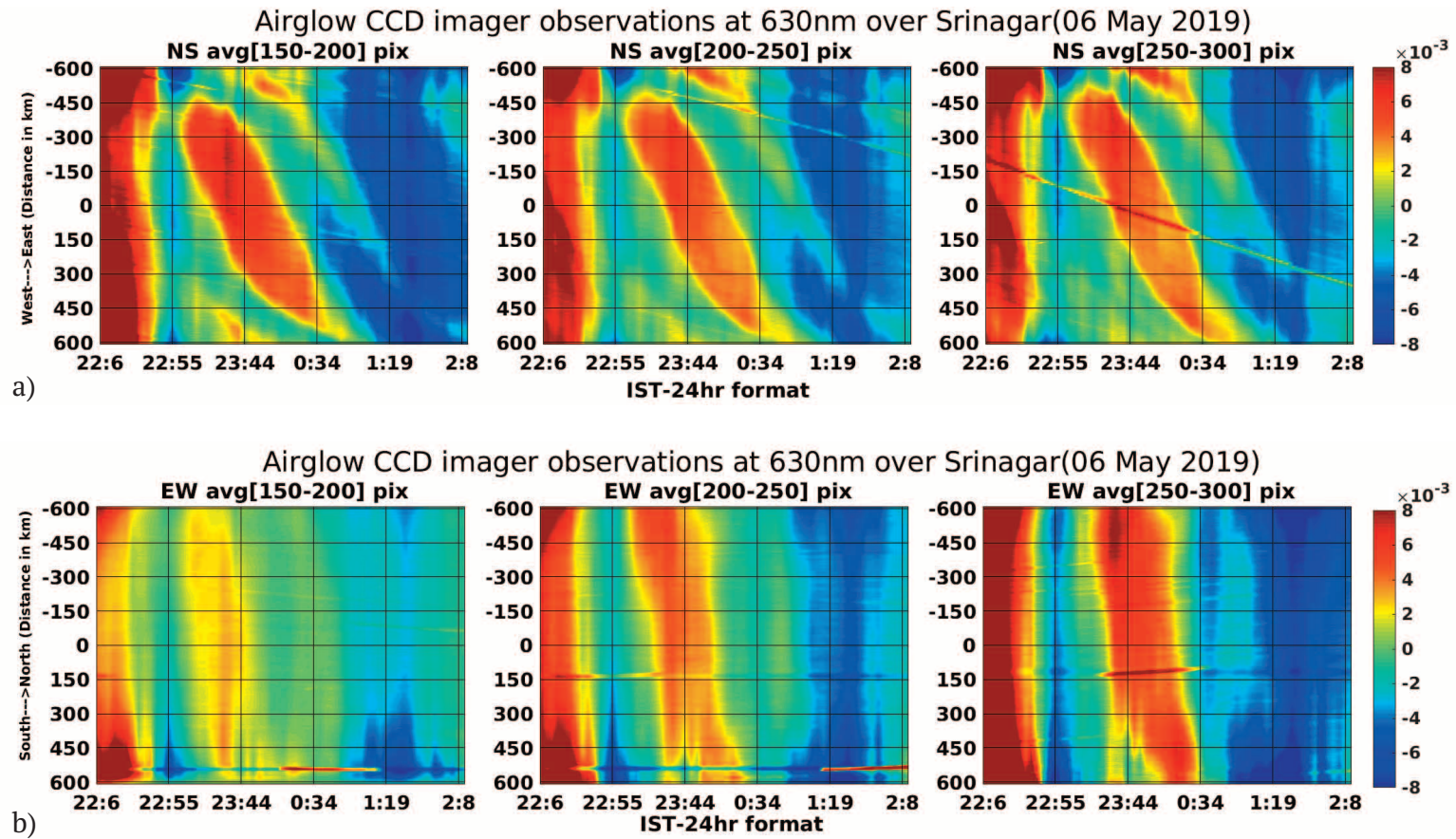

Figure 8. a) Zonal keogram of $630 \mathrm{~nm}$ airglow emissions on 06 May, 2019 and b) Meridional keogram of $630 \mathrm{~nm}$ airglow emissions on 06 May, 2019. The westward drifting bright red band seen in the keograms between 22:55 to 00:34 IST represents the plasma blob mentioned in the text.

\section{Discussion}

In this study, we report the presence of various features of mid-latitude plasma irregularities observed on two consecutive nights of May 2019 with an all sky imager. We also analyze their characteristic features and evolution processes in detail. The occurrence of field aligned irregularities over low to mid-latitude transition regions under geomagnetic quiet conditions is discussed, as well. 
As can be seen from the zonal keograms, there are strong westward disturbances going on in the form of depletion band like structures. However, dark bands parallel to the time axis can easily be seen in the meridional keogram, indicating that drift velocity has a significant contribution only in the zonal direction, i.e., these are more like N-S associated plasma depletion structures. During the two nights, seven distinct irregularites with their own morphological and other characteristics entered the all-sky imager's FOV. These irregularities are morphologically different in the sense that some are branched and some are not branched. In some cases for 05 May irregularities, detachment of the bifurcated parts of the depletion can be seen from the images. So, each irregularity has its own characteristic features associated with it. The question is whether the plasma depletions observed at latitudes $>20^{\circ}$ $\mathrm{N}$ magnetic latitudes were the extension of equatorial plasma depletions or whether they were produced locally. Sivakandan et al. [2020] has mentioned that the plasma depletion structures that appear over the low-mid latitude transition zone are difficult to classify in terms of their origin given that this region can be affected by both low and mid latitude processes in general. So, the detection of plasma depletion over such regions could be due to the equatorial plasma bubble mapping toward the low-latitudes, or it could be due to the mid-latitude irregularites extending toward the low-latitudes.

Generally, the EPBs drift eastward [Makela, 2006], while the mid-latitude spread-F structures tend to drift towards west [Sun et al., 2015]. The equatorial plasma bubble motion tends to reverse westward during geomagnetic disturbances [e.g. Abdu et al., 2003]. It's worth noting that the geomagnetic $K p$ and $A p$ indices during the nights of interest are 1 and $0+$, indicating that the measurements were made in geomagnetically quiet conditions. The data corresponding to these indices is available on the website spaceweatherlive.com. In all our cases of observed irregularities during the 05 and 06 May nights, we see the westward drifts in irregularities during geomagnetic quiet conditions. Generally, the equatorial plasma depletions deviate from the perfect N-S alignments and are observed to occur with westward tilts and increasing pole-ward latitudes [Abalde et al., 2001; Mukherjee, 2003]. In the present case, the observed irregularities are elongated in the direction of the earth's magnetic field with only smaller eastward tilts and appear in the FOV of the imager from polar side of the images. So, like the equatorial plasma depletions, the geomagnetic alignment of the observed irregularities is also not perfect but the difference is that in the present case we see eastward tilts instead of westward tilts. It's also clear from the optical images that as the equatorial depleted area rises in altitude, it often bifurcates, forming two related depleted regions [Kelley et al., 2002]. Bifurcations can also be observed in the depleted regions in the current case of irregularity structures. We see various cases of depleted regions with several finger like bifurcating structures apparent in them. In case of the equatorial depletions, the bifurcations point towards polar regions i.e. away from equator. But in our present case, the bifurcations are observed towards the lower latitude portions of the irregularities, pointing towards the equator through lower latitude regions. We attribute the bifurcation of plasma irregularities to the difference in the zonal drifts which might be arising because of secondary instabilities near low latitudes. We present the case of second irregularity structure on the 06 May night where the eastern limb bifurcates from the western limb and continue to move away from each other. The drift velocity of western limb is around $160 \mathrm{~m} / \mathrm{s}$ while the eastern limb propagates with $130 \mathrm{~m} / \mathrm{s}$, same as its stem. Because of the variations in zonal drift velocities, these bifurcations become more pronounced. Also, on the 05 May night, the eastern limb of second irregularity bifurcates solely from the main irregularity structure and gets completely detached from it. There we can again see the clear cut differences in the zonal velocities (205 m/s vs $160 \mathrm{~m} / \mathrm{s})$ of the two bifurcating structures. Normally, the spatial extension of the equatorial plasma depletions in the East-West direction ranges from $\sim 50-450 \mathrm{~km}$ [Makela, 2006]. In mid-latitude regions, airglow imaging has identified small-scale FAIs in the depleted regions of intensities of MSTIDs [Kelley et al., 2003, 2004]. FAIs associated with MSTIDs at midlatitudes usually have a scale size of the order of a few metres to a few hundred kilometers and they occur in depleted airglow regions associated with propagating MSTIDs [Sun et al., 2015]. In our observations, the longitudinal spatial extension of the branching type irregularities ranges from $\sim 140-290 \mathrm{~km}$. The branching limbs range from $\sim 80-230$ $\mathrm{km}$ in terms of longitudinal extensions. In case of non branched irregularity structures, this extension ranges from $200-468 \mathrm{~km}$. It may be noted that all the non branched irregularities are post-midnight irregularities while the branched type are post-sunset irregularities. While our observations of EW spatial extension of plasma depleted regions are consistent with equatorial plasma irregularities, such large-scale irregularities in the low to midlatitude transition regions have not been published in the literature. Thus, our observations indicate that the large scale plasma irregularities exist at the transition regions in Indian sector. Based on these observational facts, it is natural to think that the westward drifting depletion structures are not the extensions of equatorial plasma bubbles but could be the extensions of midlatitude plasma depletions towards the low midlatitudes including Srinagar. 


\section{Aashiq Hussain Bhat et al.}

To verify that the irregularities in present study are not the extensions of equatorial plasma bubbles, we looked for the GIRI (30 MHz radar) data and Digisonde observations from NARL. The nighttime F region observations through GIRI have been conducted on 06 May 2019, while it had not been operated on 05 May 2019. Coming to the digisonde observations, due to blanketing Es during nighttime, signal from F region disappeared on both the nights. The RTI (range time intensitry) map of the radar data from NARL (low latitude region in Indian sector) for the 06 May night is shown in Figure 9. From this plot, no signatures of plasma bubbles can be seen during this night near the equatorial region and as such the plasma depletions observed in the airglow images from Srinagar can not be the extensions of equatorial plasma bubbles. The depletions are present in our airglow observations from $22 \mathrm{hr}$ LT to $26 \mathrm{hr}$ LT drifting westward, and no echoing signals have been detected by the GIRI during this period, suggesting that the possibility of equatorial plasma bubbles reaching our latitudes on 06 May night can be ruled out. Since, the plasma irregularities observed during 05 May night have similar characteristics to those on 06 May night, we infer that the irregularities on both the nights are not the extensions of equatorial plasma bubbles.

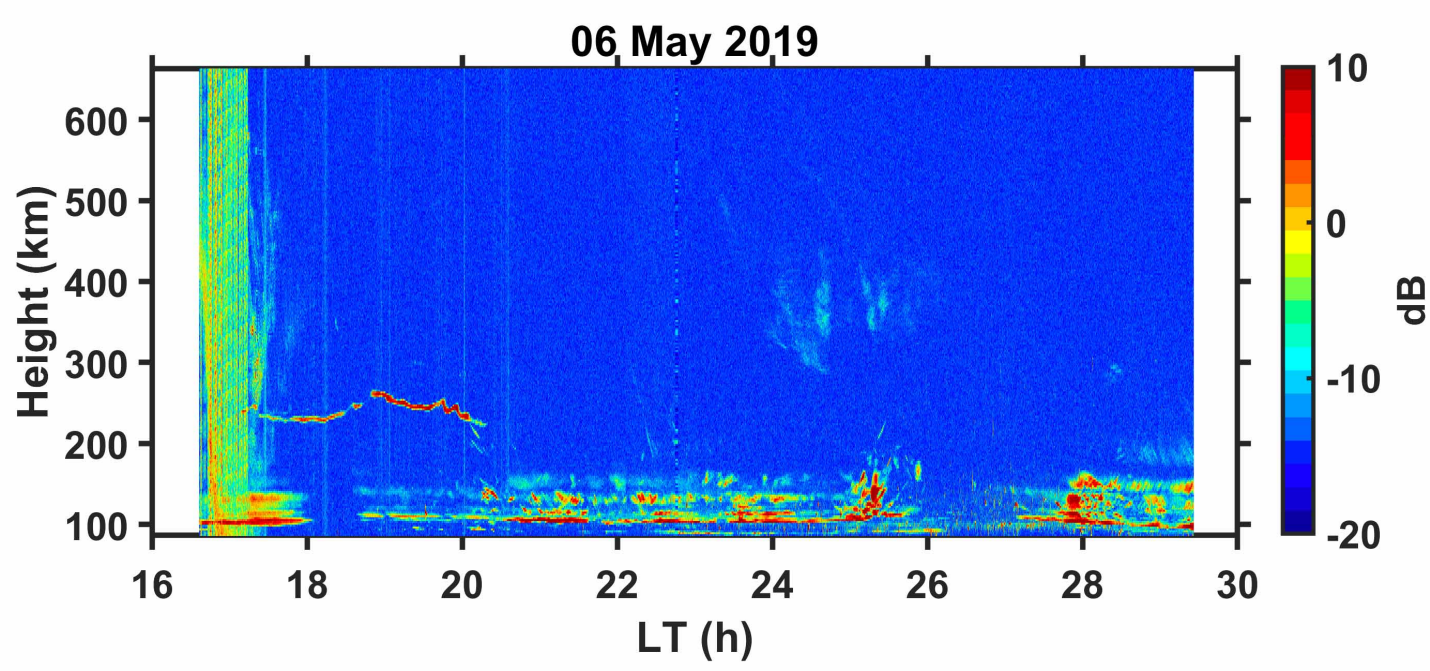

Figure 9. RTI image of radar data for the night of 06 May, 2019 over NARL (a geomagnetic low latitude station in India). The horizontal axis shows local time, while the vertical axis shows altitude.

Airglow intensity modulations can occur in the mid-latitudes through gravity waves or under the action of Perkins instability mechanism. Gravity waves from the mesosphere-lower-thermosphere region (MLT-region) ( 90$120 \mathrm{~km}$ ) might penetrate into the $\mathrm{F}$ region of the ionosphere under suitable conditions of the background atmosphere [Sivakandan et al., 2020]. So, we analysed the $557.7 \mathrm{~nm}$ and $840 \mathrm{~nm}$ images corresponding to the peak altitudes of emission i.e. 97 and $86 \mathrm{~km}$ respectively (MLT region) to check for the presence of gravity waves. Since, we see gravity wave signatures in the $840 \mathrm{~nm}$ and $557 \mathrm{~nm}$ filters during both the nights, one of the possibilities is that the observed depletions in the $630 \mathrm{~nm}$ images were under the control of upward propagating gravity wave through the airglow emission layer oscillations. The gravity waves are initially observed at $\sim 87 \mathrm{~km}$ height and shortly after their appearance at this height, these are seen in $557 \mathrm{~nm}$ filter at $\sim 97 \mathrm{~km}$ altitude. It indicates that the gravity waves are indeed propagating upwards during the $5^{\text {th }}$ and $6^{\text {th }}$ May nights. As an example, we have shown the presence of mesospheric gravity waves during these nights in Figure 10. These waves are propagating mostly in the North-East direction with phase fronts along NW-SE direction which doesn't match with the propagation direction of the observed plasma irregularities. Thus, these gravity waves are not directly observed in the ionospheric heights but could have still influenced the plasma density at ionospheric levels through secondary wave generation. The F-region plasma is transported to higher altitudes by the northward and upward plasma drift while it is transported to lower altitudes by the southward and downward plasma drift [Otsuka et al., 2007]. The emission rate of airglows depends directly on the product of $\mathrm{O}_{2}$ and $\mathrm{O}+$ densities and as a result it induces a reduction or increase in airglow strength depending on the upward or downward plasma drift respectively. 
The other possiblity of generation of the large scale plasma depletions can be through the Perkins' instability mechanism, but its growth rate to generate such irregularites is generally less. Although the growth rate of Perkins' instablity could be less yet the gravity wave seeding can be helpful in exciting the large-scale plasma instabilities in the midlatitude ionosphere through this instability [Hamza, 1999]. However, to fully comprehend the physical processes of FAI generation mentioned in this paper, more observational evidences and other theoretical studies are needed.



Figure 10. Gravity waves detected in the MLT region on both the 5th and 6th of May, 2019. The waves are seen at $\sim 87 \mathrm{~km}$ height via $840 \mathrm{~nm}$ OH interference filter and with a short delay at $\sim 97 \mathrm{~km}$ height through $557 \mathrm{~nm}$ interference filter indicating their upward propagation.

Finally, we highlight the presence of post-midnight MSTIDs in the $630 \mathrm{~nm}$ images during the night of May 05, which appeared as wave-like perturbation structures. Figure 5 depicts the spatial structures of MSTID in the Fregion and FAI from 23:49 to 01:23 IST, when MSTIDs are more intense. MSTIDs extend from the northwest to the southeast and propagate to the southwest. The periodic airglow enhancement and depletions caused by the MSTID are observed to be connected with the FAIs. It is observed that the wavelength of the MSTID is exactly comparable to the inter-depletion distance of the FAIs observed at that time and to which the MSTID structures seem to be connected. Moreover, these two phenomena propagate together. Based on these reasons, we infer that their visual connection from the airglow images can be assumed to be their actual connection which suggests that the MSTID is coupled with F region FAI along the geomagnetic field lines. Our results hint at a possibility that the post-midnight MSTIDs can be seeded by FAIs. The exact mechanism of how these FAIs could generate MSTIDs near the lower midlatitudes demands further rigorous investigation. 


\section{Aashiq Hussain Bhat et al.}

\section{Summary and Conclusion}

The morphological characteristics of different ionospheric irregularity events, as well as one of the post-midnight MSTID events, are presented in this study. More importantly, the occurrence of this MSTID simultaneously with the FAIs that were observed by an all-sky imager, in the campus of KU Srinagar, a low mid-latitude station, during the nights of 05 and 06 May 2019. The following is a summary of the key findings:

Post-sunset FAIs are reported for the first time from our location (geomagnetic transition region). The observed FAIs are large-scale structures and continuously propagate westward from eastern regions. During the two nights, about seven distinct plasma depletion structures with their own morphological and other characteristics entered the all-sky imager's FOV. In all the cases of observed irregularities we observe the westward drifts implying that the mid-latitude FAIs reaching the transition region also drift westward.

The full evolution, amplification and dissipation of these large scale $(>250 \mathrm{~km})$ and some meso-scale FAIs $(\sim 140$ $\mathrm{km})$ in the airglow images were observed through the imager. These large scale FAIs together with an MSTID occurred under geomagnetically quiet conditions (Kp 1) indicating that the mid-latitude irregularities with large scales also exist and can reach the low latitude transition region even during the geomagnetic quiet conditions.

One interesting observation is that the gravity waves are seen in the MLT region simultaneously with the FAIs during both nights. The gravity waves from below might be an influencing factor to generate such large scale plasma irregularities in the presented cases. However, the physical mechanism(s) governing the FAI propagation remain unknown, and further empirical evidence and other theoretical studies are required to fully comprehend the physical processes of FAI generation discussed in this paper.

The evolution and properties of the post-midnight MSTID event near lower latitudes were most likely regulated by the FAIs as seen from the airglow observations.

The main features of the FAIs (believed to be mid-latitude generated) in the present case are similar to the FAIs corresponding to equatorial regions in certain respects like the East-west extensions, zonal drift velocities and the bifurcation/tree like branching structures. The differences are that the equatorial plasma depletions, mostly propagating eastward, have bifurcations towards higher latitude side and exist with westward tilts while the irregularities as seen in the present case propagating westward, have bifurcations towards lower latitude side and exist with eastward tilts. No EPBs have been observed near the equatorial regions during the two nights under consideration. Thus, we infer that such irregularities are likely generated in mid-latitude region and extend towards the geomagnetic transition region. The observations are being continuously taken during clear sky, moonless nights to collect further details of such features in the ionosphere over Srinagar such that the mechanisms responsible for their generation and dissipation are known and the generalized properties are verified.

Acknowledgement. Aashiq Hussain Bhat thanks DST while Bilal Ahmad Ganaie thanks UGC for financial supports. We thank NARL for allowing the use of their facilities and installation of Airglow Imager at Kashmir University, Srinagar. We are also grateful to the NOAA and Kyoto teams for making geomagnetic indices data available on the internet through the website spaceweatherlive.com.

\section{References}

Abalde, J., P. Fagundes, J. Bittencourt, and Y. Sahai (2001). Observations of equatorial F region plasma bubbles using simultaneous OI $777.4 \mathrm{~nm}$ and OI $630.0 \mathrm{~nm}$ imaging: New results, J. Geophys. Res., 106, 30,331-30,336, doi:10.1029/2001JA001115.

Abdu, M.A., I.S. Batista, H. Takahashi, J. MacDougall, J.H. Sobral, A.F. Medeiros, and N.B. Trivedi (2003). Magnetospheric disturbance induced equatorial plasma bubble development and dynamics: A case study in Brazilian sector, J. Geophys. Res., 108, A12, 1449, doi:10.1029/2002JA009721.

Bhat, A.H., Ganaie, B.A., Ramkumar, T.K., Chaitanaya P.P., Malik M.A. (2021). Simultaneous detection of mediumscale traveling ionospheric disturbances and ionospheric plasma irregularities over Srinagar, J\&K, India. Acta Geophys., https://doi.org/10.1007/s11600-021-00590-w

Bowman, G.G., \& Monro, P.E. (1988). Mid-latitude range spread and travelling ionospheric disturbance, J. 
Atmos.Terres. Phys., 50, 3, 215-223, https://doi.org/10.1016/0021-9169(88)900700.

Garcia, F.J., M.J. Taylor and M.C. Kelly (1997). Two-dimensional spectral analysis of mesospheric airglow image data, Appl. Opt., 36, 7374-7385.

Garcia, F.J., M.C. Kelley, J.J. Makela, and C.-S. Huang (2000). Airglow observations of mesoscale low-velocity traveling ionospheric disturbances at midlatitudes, J. Geophys. Res., 105, 18,407-18,415, https://doi.org/10.1029/1999JA000305.

Hamza A.M. (1999) Perkins instability revisited, J. Geophys. Res., 104, A10, 22,567-22,575.

Huang, C.S., J.C. Foster and Y. Sahai (2007). Significant depletions of the ionospheric plasma density at middle latitudes: A possible signature of equatorial spread F bubbles near the plasmapause, J. Geophys. Res., 112, A05, 315. https://doi.org/10.1029/2007JA012307.

Kelley, M.C., J.J. Makela, L.J. Paxton, F. Kamalabadi, J.M. Comberiate, and H. Kil (2003). The first coordinated groundand space-based opticalobservations of equatorial plasma bubbles, Geophys. Res. Lett., 30, 14, 1766, doi:10.1029/2003GL017301.

Kelley, M. C., W. E. Swartz and J. J. Makela (2004). Mid-latitude ionospheric fluctuation spectra due to secondary E $\times$ B instabilities, J. Atmos, Solar-Terres. Phys., 66, 17, 1559-1565, https://doi.org/10.1016/j.jastp.2004.07.004.

Kelley, M.C. (2011). On the origin of mesoscale TIDs at midlatitudes, Annales Geophysicae, 29, 2, 361-366, https://doi.org/10.5194/angeo.

Kubota, M., H. Fukunishi, S. Okano (2001). Characteristics of medium- and large- scale TID over Japan derived from OI 630 nm nightglow observation, Earth Planets Space, 53, 741751.

Ma, G., and T. Maruyama (2006). A super bubble detected by dense GPS network at east Asian longitudes, Geophys. Res. Lett., 33, L21103, doi:10.1029/2006GL027512.

Makela, J.J., (2006). A review of imaging low-latitude ionospheric irregularity processes, J. Atmos. and Sol. Terr. Phys., 68, 1441-1458.

Martinis, C., J. Baumgardner, J. Wroten and M Mendillo (2010). Seasonal dependence of MSTIDs obtained from 630.0 nm airglow imaging at Arecibo, Geophys. Res. Lett., 37, L11103, https://doi.org/10.1029/2010GL043569.

Mukherjee, G.K. (2003), Studies of the equatorial F-region depletions and dynamics using multiple wavelength night glow imaging, J. Atmos. Sol. Terr. Phys., 65, 379-390.

Narayanan et al. (2009). Imaging observations of upper mesospheric nightglow emissions from Tirunelveli(8.7o N), Indian J. Radio Space Phys., 38, June 2009, 150-158.

Narayanan, V.L., K. Shiokawa, Y. Otsuka, and S. Saito (2014). Airglow observations of nighttime medium-scale traveling ionospheric disturbances from Yonaguni: Statistical characteristics and low-latitude limit, J. Geophys. Res.: Space Physics, 119, 9268-9282, https://doi.org/10.1002/2014JA020368.

Otsuka, Y., K. Shiokawa, T. Ogawa, T. Yokoyama, M. Yamamoto, S. Fukao (2004). Spatial relation of equatorial plasma bubbles and field aligned irregularities observed with an all-sky airglow imager and the equatorial atmosphere radar, Geophys. Res. Lett., 31, L20802, doi:10.1029/2004GL020869.

Otsuka Y., F. Onoma, K. Shiokawa T. Ogawa, M. Yamamoto and S. Fukao (2007). Simultaneous observations of nighttime medium-scale traveling ionospheric disturbances and E region field-aligned irregularities at midlatitude, J. Geophys. Res., 112, A06317, doi:10.1029/2005JA011548.

Otsuka Y, K. Shiokawa, T. Ogawa T. Yokoyama and M. Yamamoto (2009). Spatial relationship of nighttime mediumscale traveling ionospheric disturbances and F region field-aligned irregularities observed with two spaced allsky airglow imagers and the middle and upper atmosphere radar, J. Geophys. Res., 114 A05302, doi: 10.1029/2008JA013902.

Otsuka Y, K. Shiokawa, M. Nishioka and Effendy (2012).VHF radar observations of post-midnight F-region fieldaligned irregularities over Indonesia during solar minimum, Indian J. Radio Space Phys., 41, 199-207.

Patra, A.K., P. Srinivasulu, P.P., Chaitanya, M.D. Rao and A. Jayaraman (2014). First results on low-latitude E and F region irregularities obtained using the Gadanki Ionospheric radar interferometer, J. Geophys. Res.: Space Physics, 119, 10,276-10,293, https://doi.org/10.1002/2014JA020604.

Ramkumar, T.K., Malik, M.A., Ganaie, B.A., Bhat, A.H., (2021). Airglow imager based observation of possible influences of subtropical mesospheric gravity waves on F region ionosphere over Jammu \& Kashmir, India. Sci. Rep. https://doi.org/10.1038/s41598-021-89694-3.

Shiokawa, K., C. Ihara, Y. Otsuka, and T. Ogawa (2003). Statistical study of nighttime medium-scale traveling ionospheric disturbances using midlatitude airglow images, J. Geophys. Res., 108, A1, 1052, 


\section{Aashiq Hussain Bhat et al.}

doi:10.1029/2002JA009491.

Saito, S., M. Yamamoto and H. Hashiguchi (2008). Imaging observations of nighttime mid-latitude F-region field-aligned irregularities by an MU radar ultra-multi-channel system, Annales Geophysicae, 26, 8, 23452352, https://doi.org/10.5194/angeo-26-2345-2008.

Sivakandan, M., D. Chakrabarty, T.K. Ramkumar, A. Guharay, A. Taori, and N. Parihar (2019). Evidence for deep ingression of the midlatitude MSTID into as low as $\sim 3.5^{\circ}$ magnetic latitude, J. Geophys. Res.: Space Physics, 124, 749-764, https://doi.org/10.1029/2018JA026103.

Sivakandan, M., S. Mondal, S. Sarkhel, D. Chakrabarty, M.V. Sunil Krishna, P.P. Chaitanya, A.K. Patra, R.K. Choudhary, T.K. Pant, A.K. Upadhayaya, Takuya Sori (2020). Mid-latitude spread-F structures over the geomagnetic low-mid latitude transition region: An observational evidence. J. Geophys. Res.: Space Physics, 124, e2019JA027531, https://doi.org/10.1029/2019JA027531.

Sun, L., J. Xu, W. Wang, X. Yue, W. Yuan, N. Ning, D. Zhang, F.C. de Meneses (2015). Mesoscale field-aligned irregularity structures (FAIs) of airglow associated with medium-scale traveling ionospheric disturbances (MSTIDs), J. Geophys. Res.: Space Physics, 120, 9839-9858, https://doi.org/10.1002/2014JA020944.

Taori, A., N. Parihar, R. Ghodpage, N. Dashora, S. Sripathi, E.A. Kherani and P.T. Patil (2015). Probing the possible trigger mechanisms of an equatorial plasma bubble event based on multistation optical data, J. Geophys. Res.: Space Physics, 120, 8835-8847, https://doi.org/10.1002/2015JA021541. 